\title{
Medical Imaging Engineering and Technology Branch of the Chinese Society of Biomedical Engineering expert consensus on the application of Emergency Mobile Cabin CT
}

\author{
Qi Yang ${ }^{1}$, Haibo $\mathrm{Xu}^{2}$, Xiaoying Tang ${ }^{3}$, Chunhong $\mathrm{Hu}^{4}$, Peijun Wang ${ }^{5}$, Yì Xiáng J. Wáng ${ }^{6}$, Yaofa Wang ${ }^{7}$, \\ Guolin $\mathrm{Ma}^{8}$, Bing Zhang'; on behalf of the Expert Committee of Medical Imaging Engineering and \\ Technology Branch of the Chinese Society of Biomedical Engineering*
}

${ }^{1}$ Beijing Chaoyang Hospital, Capital Medical University, Beijing, China; ${ }^{2}$ Zhongnan Hospital of Wuhan University, Wuhan, China; ${ }^{3}$ Beijing Institute of Technology, Beijing, China; ${ }^{4}$ The First Affiliated Hospital of Soochow University, Soochow, China; ${ }^{5}$ Tongji Hospital of Tongji University, Shanghai, China; ${ }^{6}$ Department of Imaging and Interventional Radiology, The Chinese University of Hong Kong, Hong Kong, China; ${ }^{7}$ Minfound Medical Systems Co. Ltd, Shaoxing, China; ${ }^{8}$ China-Japan Friendship Hospital, Beijing, China; ${ }^{9}$ The Affiliated Drum Tower Hospital of Nanjing University Medical School, Nanjing, China

Correspondence to: Guolin Ma. Department of Radiology, China-Japan Friendship Hospital, No. 2 East Yinghua Road, Chaoyang District, Beijing 100029, China. Email: maguolin1007@qq.com; Bing Zhang. The Affiliated Drum Tower Hospital of Nanjing University Medical School, Nanjing 210008, China. Email: zhangbing_nanjing@vip.163.com.

\begin{abstract}
Started during December 2019, following the emergence of several COVID-19 cases in Wuhan City, Hubei Province, there was a rapid surge and spread of new COVID-19 cases throughout China. The disease has since been included in the Class B infectious diseases category, as stipulated in the Law of the People's Republic of China on the Prevention and Treatment of Infectious Diseases and shall be managed according to Class A infectious diseases. During the early phases of COVID-19 infection, no specific pulmonary imaging features may be evident, or features overlapping with other pneumonia may be observed. Although CT is not the gold standard for the diagnosis of COVID-19, it nonetheless is a convenient and fast method, and its application can be deployed in community hospitals. Furthermore, CT can be used to render a suggestive diagnosis and evaluate the severity as well as the effects of therapeutic interventions for typical cases of COVID-19. The mobile emergency special CT device described in this document (also known as Emergency Mobile Cabin CT) has several unique characteristics, including its mobility, flexibility, and networking capabilities. Furthermore, it adopts a fully independent isolation design to avoid cross-infection between patients and medical staff. It can play an important role in screening suspected cases presenting with imaging features of COVID-19 in hospitals of various levels that provide care to suspected or confirmed COVID-19 patients as part of the first line procedures of epidemic prevention and control.
\end{abstract}

Keywords: Emergency mobile cabin CT; COVID-19; cross-infection exposure

Submitted Aug 17, 2020. Accepted for publication Aug 31, 2020.

doi: 10.21037/qims-20-980

View this article at: http://dx.doi.org/10.21037/qims-20-980

\footnotetext{
* Expert Committee: Xiaoying Tang, Chunhong Hu, Yaofa Wang, Peijun Wang, Xiangxing Ma, Guolin Ma, Haibo Xu, Bing Zhang, Haochuan Jiang, Yun Zheng, Chuandong Li, Qi Yang, Jiangyong Luo, Xiwei Zhang, Yadong Gang, Xiaochun Zhang, Fang Zheng, Qinglian Guo, Chunhua Chai, Liming Guan, Hong Gao, Bo Gao, Ruiwang Huang, Huijie Jiang, Bin Lv, Jie Lu, Jingxin Liu, Haimei Li, Ming Li, Hong Lu, Shanxing Ou, Haibin Shi, Xiaochun Wang, Jian Wang, Xiaoyan Xin, Jianhua Yuan, Hongwei Yu, Heping Zhou, Xin Zhang.
} 


\section{Equipment of emergency mobile cabin CT}

\section{Name and structure of emergency mobile cabin CT}

Emergency mobile cabin CT, a special emergency CT equipment, is a solution conceptualized and developed in China as part of the primary response to the ever-evolving coronavirus disease (COVID-19) pandemic. The system is composed of a CT equipment, a radiation protection unit, an independent operating room, an independent scanning room (equipped with an electric door), network communication systems, air conditioning, secure ultraviolet air disinfection facilities, power distribution system, ventilation, etc. It has numerous unique features, including mobility, network accessibility, independent isolation, quick outdoor installation, minimized risk of cross-infection, quick examination turnover, etc. It is composed of a CT scan and a diagnostic unit that can be independent of the departments of Radiology or Medical Imaging.

The front view and top view of the emergency mobile cabin CT are shown in Figure 1.

\section{The layout of emergency mobile cabin $C T$}

A three-dimensional rendering of emergency mobile cabin CT is shown in Figure 2.

\section{Advantages of emergency mobile cabin CT compared with other CT scanning modalities}

Advantages of emergency mobile cabin CT compared with conventional CT scanning

\section{Flexible mobility}

The emergency mobile cabin CT is designed such that the transportation shock-absorption system in the cabin (1) can withstand the impact-load generated by variable driving conditions during transportation, and can also provide protection for the precision parts of the CT system, such as the bearings, slip rings, $\mathrm{X}$-ray tubes, etc., under adverse road conditions. The emergency mobile cabin CT has high flexible mobility during long-distance transportation and quick site transfer when hauled by ordinary trucks.

\section{The capability of rapid deployment}

The emergency mobile cabin CT is a complete set of CT clinical solutions. Compared with conventional CT, it does not need to be installed and debugged on-site. Instead, all components are installed and debugged prior to leaving the factory. It is equipped with an independent air conditioning unit, an exhaust system, systematic protection against ionizing radiation, and it can be rapidly deployed on-site and mobilized for use when powered on.

\section{Better adaptability to the environment}

The emergency mobile cabin CT does not need an indoor environment and does not take up the hospital room resources. It contains a rainproof, earthquake resistant, heat insulated and sterile environment, and can also adapt to various outdoor environments and application scenarios, such as the emergency needs of the outbreak, earthquake and other natural disasters. It can support field rescue operations thanks to its flexible mobility and rapid deployment capability.

\section{Prevention of cross-infection}

With intelligent electrical door design and voice communication function, an intelligent isolated positioning system fully realizes the remote positioning of the patient. The intelligent natural ventilation system in the scanning room is superior to that in the conventional CT room, which circumvents the common shortcomings of conventional CT scanning rooms.

\section{Special disinfection system}

The scanning room and the operation room are equipped with ultraviolet (UV) disinfection facilities, which can carry out comprehensive disinfection without leaving a dead corner and can achieve disinfection for every patient.

Independence, isolation, and mobility

The emergency mobile cabin CT is a CT scanning unit independent of the Radiology or Imaging department of the hospitals, which is placed outdoors and meets the requirements of the isolation examination unit during the epidemic (2). The isolation design of the scanning room and the operating room offers several advantages. The emergency mobile cabin CT can be moved and used in another hospital at any time. It can also be installed in the hospital and be incorporated as a standard equipment of the Radiology department. Therefore, the emergency mobile cabin CT can be utilized in multiple situations.

\section{Application of $5 G$ network}

The mobile cabin $\mathrm{CT}$ is equipped with $5 \mathrm{G}$ network communication, which incorporates the scanning unit as a part of the network system. It can be connected with picture archiving and communication systems (PACS), hospital information system (HIS), and radiology information system (RIS) of the hospital, and the massive image data can be transmitted to these units. At the output terminal, it allows data access for efficient post-processing and enables independent assessment and diagnosis by other experts connected to the network. 


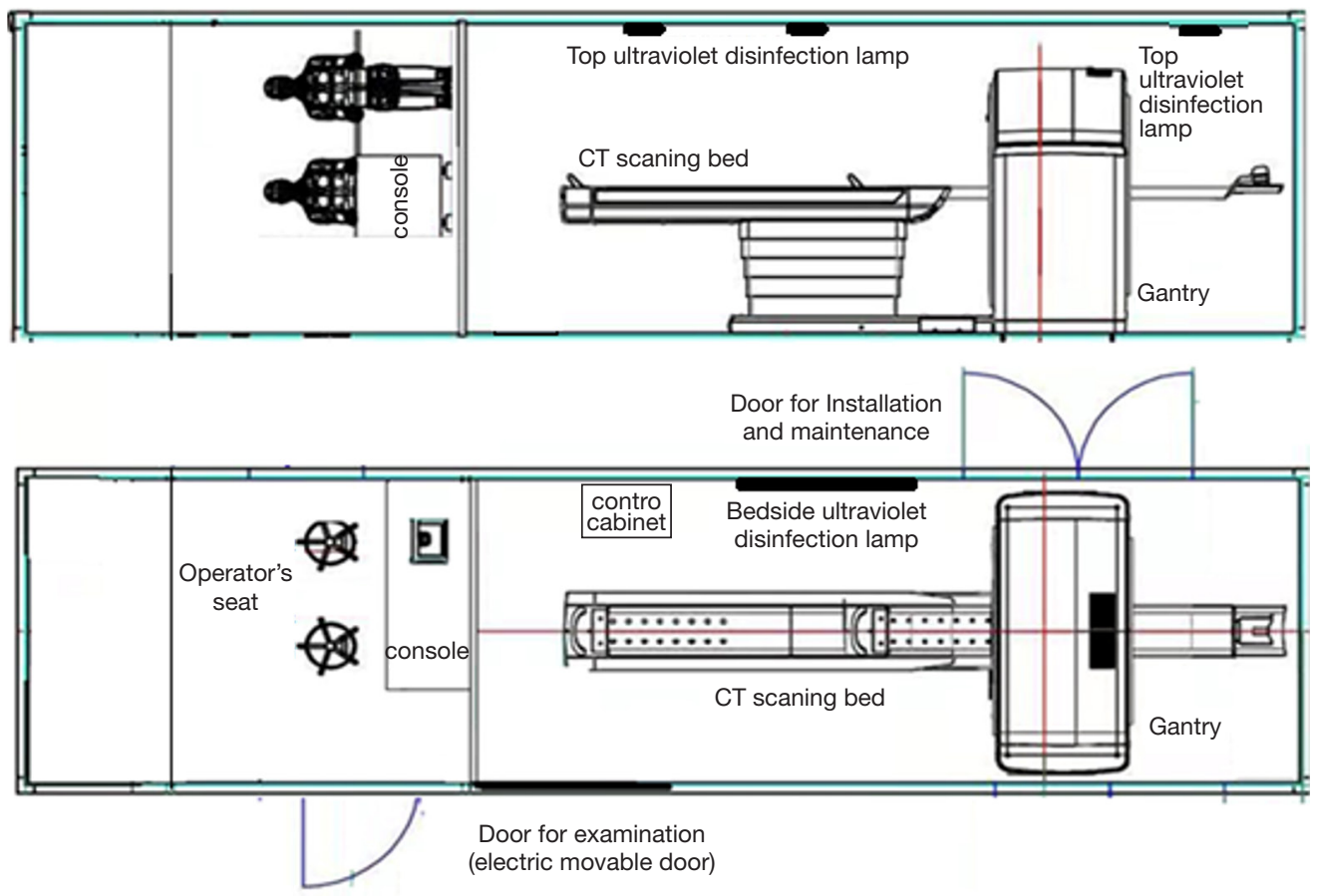

Figure 1 The front and top views of the emergency mobile cabin CT.

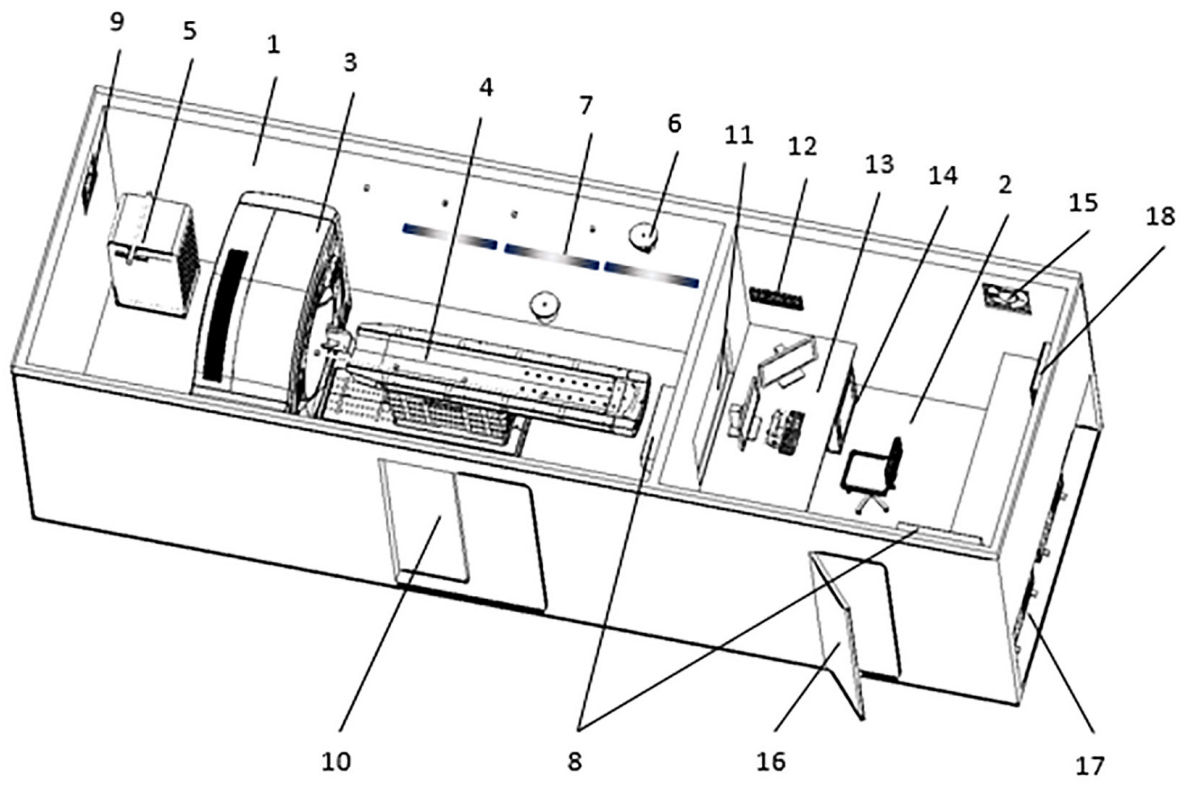

Figure 2 Three-dimensional stereogram of emergency mobile cabin CT. 1: scanning room; 2: operating room; 3: CT system; 4: scanning bed; 5: power distribution unit (PDU); 6: camera; 7: ultraviolet lights; 8: air conditioner internal unit; 9: protective shutters; 10: electric movable door (scanning room); 11: lead glass observation window; 12: voltage indicator; 13: operating table; 14: the console host; 15: ventilator; 16: door (operating room); 17: air conditioner external unit; 18: consumer unit. 
Table 1 Emergency mobile cabin CT vs. vehicle CT

\begin{tabular}{|c|c|c|c|}
\hline Content & Emergency Mobile cabin CT & Vehicle CT (Truck) & Vehicle CT (Passenger vehicle) \\
\hline Patient access & No need for auxiliary steps & Auxiliary steps needed & Auxiliary steps needed \\
\hline Barrier-free channel & Available and convenient & Need additional or manual handling & Need additional or manual handling \\
\hline Stability of CT operation platform & High & Low & Low \\
\hline $\begin{array}{l}\text { Air-conditioning system for } \\
\text { temperature and humidity control }\end{array}$ & Available & Available & Available \\
\hline Expanding space & Available & Unavailable & Unavailable \\
\hline UPS of console & Available & Available & Available \\
\hline Disinfection facility & Available & Unavailable & Unavailable \\
\hline Modification period & Fast & Slow & Slow \\
\hline $\begin{array}{l}\text { The electric door of the scanning } \\
\text { room }\end{array}$ & Available & Unavailable & Unavailable \\
\hline
\end{tabular}

Advantages and disadvantages of emergency mobile cabin CT compared with other CTs installed on a Vehicle

Compared with CTs installed on a Vehicle (vehicle CT), the emergency mobile cabin CT has many characteristics that render it more widely applicable, which include spacious patient passageway, barrier-free patient access without auxiliary steps, independent isolation channel. Specific details are shown in Table 1.

\section{Advantages of emergency mobile cabin CT in practical application}

Full scope and flexible application: with its mobility, flexibility, efficiency, and network capabilities, emergency mobile cabin CT can play an important role in hospital fever clinics, emergency and critical care departments (hospital epidemic prevention and control units), mobile cabin hospitals, community epidemic screening sites, national defense medical equipment support, earthquake disasters, and other medical emergencies. The emergency mobile cabin CT has the following applicable features.

\section{Simple power on and immediate use}

The cabin CT can be operational after a simple power-on as long as the site is provided with a $380 \mathrm{~V} / 80 \mathrm{~kW}$ power supply. This quick deployment can avoid the need for the lengthy design and installation of a conventional scanning room.

\section{Fully independent isolation design to avoid cross- infection}

The operation room and the scanning room are separated, and the access for medical staff and examinees are independent.

Artificial intelligence (AI)-enabled intelligent positioning and isolated scanning: medical technicians do not have to enter the scanning room.

\section{Timely disinfection system}

Equipped with strong ultraviolet air disinfection facilities and a timely "scanning-disinfection-scanning" procedure, the system enables one-to-one disinfection and prevents cross-infection between medical staff and patients. It 

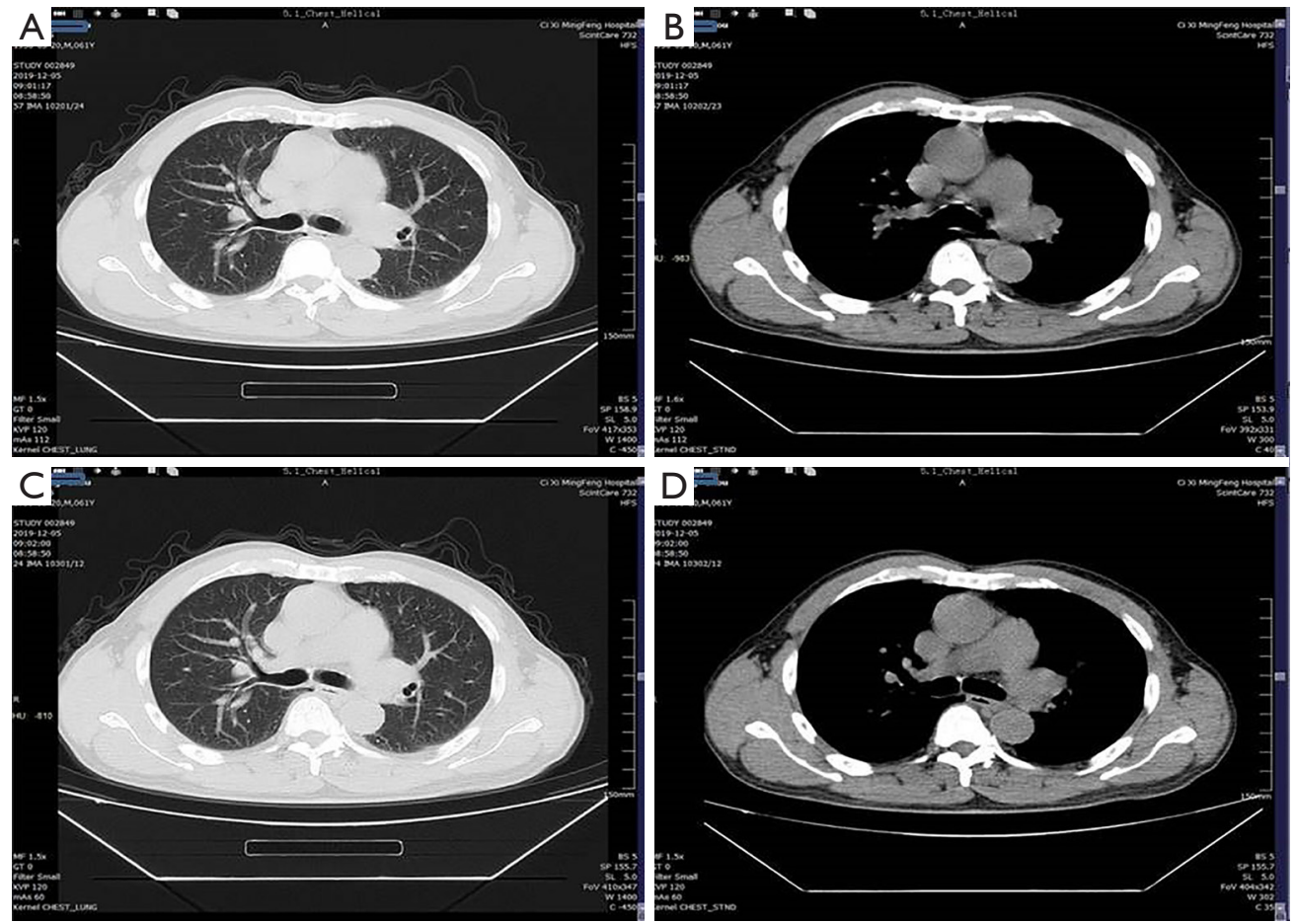

Figure 3 CT Chest image comparison at different radiation doses in the same volunteer subject. The upper panels represent the conventional dose $(120 \mathrm{kV}, 110 \mathrm{mAs})$ and the lower panels are the NDI reconstruction at a low dose $(120 \mathrm{kV}, 60 \mathrm{mAs})$.

can effectively cut off the potential transmission route when performing CT examinations on patients with highly infectious diseases, such as COVID-19 and SARS pneumonia. The ultraviolet air disinfection is proven effective for epidemic prevention and control.

\section{Higher resolution image acquisition}

The high resolution image mode of $1,024 \times 1,024$ display can clearly show the minute density changes of ground-glass lesions, which is beneficial to the density analysis of small lesions and facilitates timely diagnosis (3). If combined with specific diagnostic methods, such as nucleic acid-based polymerase chain reaction (PCR) approaches, the proposed approach can be a very effective approach for early screening of novel coronavirus infections. Furthermore, high resolution imaging is pratical for evaluating prognosis accurately and planning appropriate therapeutic strategies in suitable patients (4).

\section{Low-dose intelligent reconstruction}

Using an intelligent milliamp technology referred to as "imA", the system can automatically control the output $\mathrm{mA}$ of the tube according to the patient's body shape and the scan volume, thereby effectively reducing the radiation dose accordingly (5). The radiation dose can be reduced by approximately $30 \%$, while maintaining the same image quality.

The Nano-Dose Iterative (NDI) micro-dose iteration technology and "dual-domain" iterative algorithm are adopted, which is the iterative reconstruction algorithm based on the "projection domain" and "image domain" of the physical model (6). The algorithm can significantly reduce the CT scan dose (typical lung scan conditions can be reduced from the original 110 to $60 \mathrm{mAs}$; the dose reduction percentage can reach $45 \%$ ) (Figure 3).

The dual low-dose technology is particularly suitable for the early detection and diagnosis of COVID-19, which 


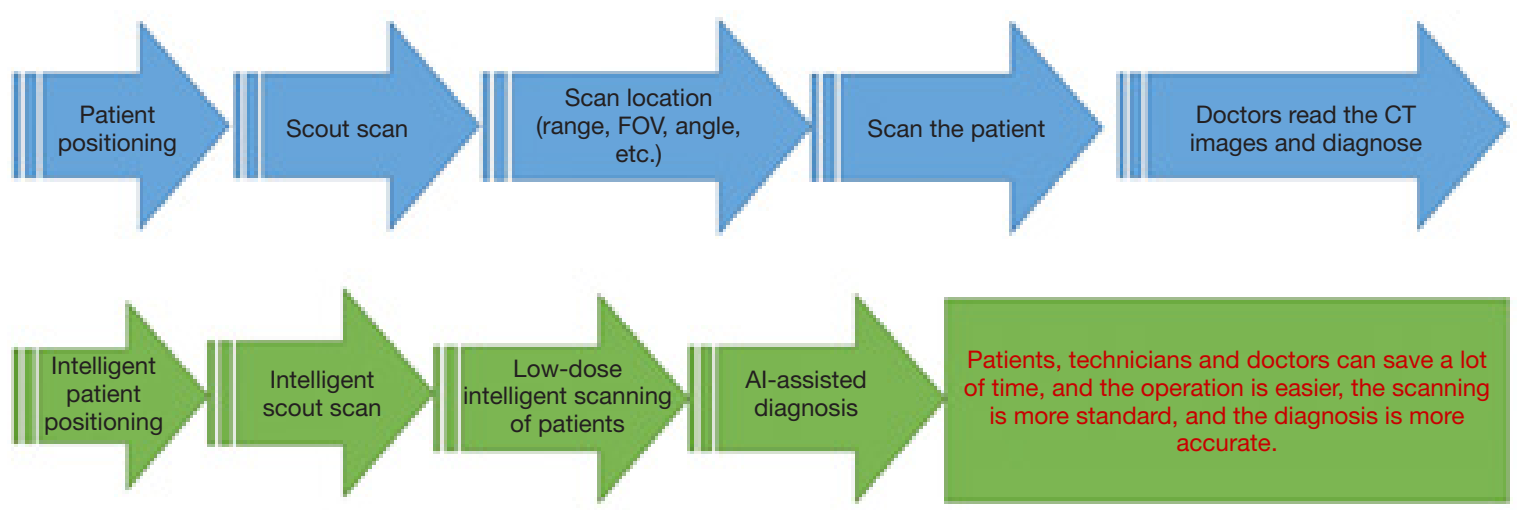

Figure 4 Intelligent scanning process versus conventional scanning process.

contributes to the health of patients who may receive multiple CT scans in a short time, avoiding excessive radiation damage and the potential carcinogenic risk associated with high doses of radiation exposure.

\section{Intelligent scanning process}

After the patient enters the scanning room, automatic patient positioning, original location, and an intelligent and personalized scanning protocol selection will be carried out, after that the scanned images will be intelligently detected to assist doctors and technicians in completing the scanning task with optimal quality and speed. The intelligent scanning process is shown in the following figure (Figure 4).

\section{Intelligent automatic positioning}

Depending on the physiological information of the patient, such as height and weight, the system will automatically select the starting and ending location of the scout view and instruct the patient accordingly through the voice dialogue system (such as to inform the patient to reposition their posture, etc.), which circumvents the need for direct contact with the patient while carrying out the patient positioning and scout scanning simultaneously.

\section{Intelligent location selection}

On the basis of the scout view image, deep learning algorithms (such as YOLO, etc.) can be utilized to perform intelligent positioning and FOV intelligent selection of head, chest, abdomen and other body parts, to quickly and accurately determine the patient's scanning part, reduce the manual operating time of the technician, minimize human error, and decrease extra radiation caused by scanning unnecessary parts (Figure 5).

\section{Intelligent scanning plan}

According to the height, weight, age, and previous medical history of the patient, the matching scanning protocol will be automatically selected to reduce the scanning dose as much as possible and to reduce the operation time of the technician, while also ensuring image quality.

\section{Intelligent detection of lesions}

"The COVID-19 AI", the intelligent detection software, is integrated with the mobile cabin CT system and AI is used to rapidly screen patients and exclude suspected cases (7). An accurate diagnosis of pneumonia, in terms of classification and stage of infection with a structured report, and the quantitative analysis and therapeutic effect evaluation of pneumonia development will be carried out by synthesizing many CT scan images obtained at several times so that the diagnosis workload of doctors will be significantly reduced. The diagnosis efficiency and accuracy will be improved.

\section{Data network transmission and remote diagnosis}

For hospitals that lack radiologists or individuals trained to render an accurate diagnosis, the remote auxiliary diagnosis module can be used to upload images in realtime, therefore, carry out intelligent detection, or render remote diagnosis/consultation, reduce patient waiting time and speed up diagnosis. The image(s) can also be sent to the Radiology department PACS or the remote consultation center, which can meet the needs of different applications at 


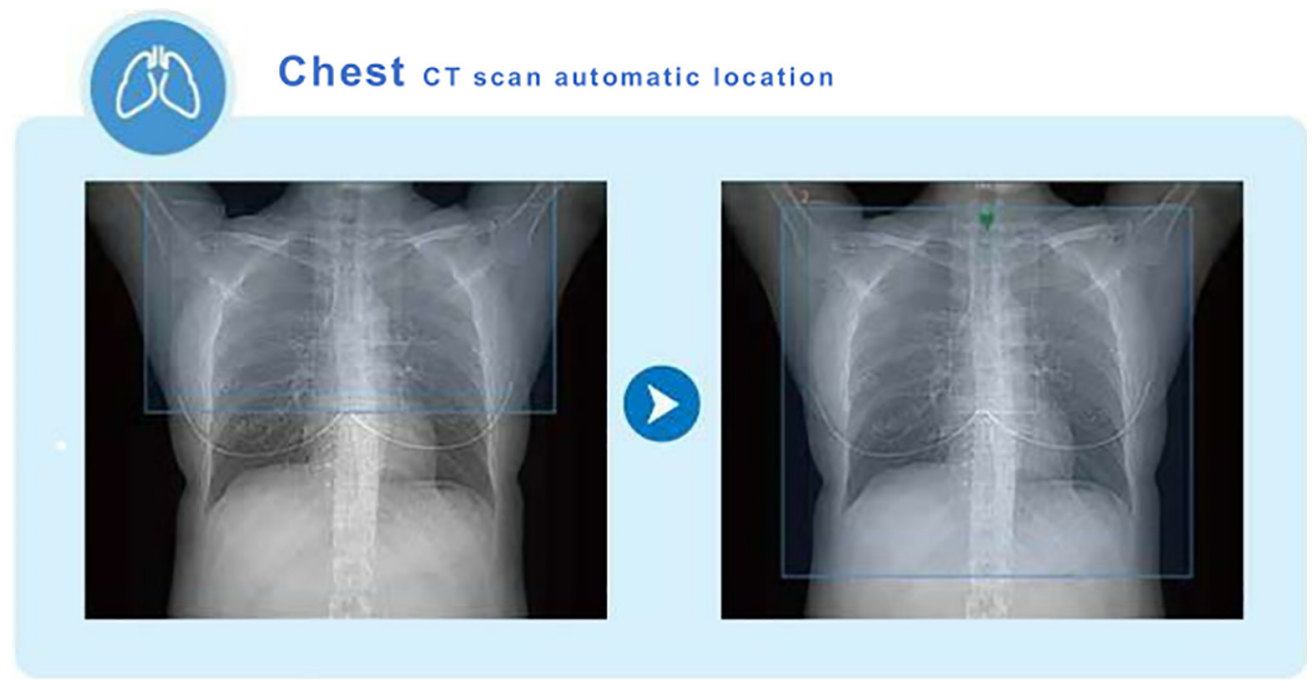

Figure 5 Intelligent chest CT scan location (the blue box in this figure is FOV).

any time. It is of great value in public emergencies, during earthquake relief operations, in field rescue missions, and similar emergency circumstances.

\section{Application of mobile cabin CT}

\section{Application scope of mobile cabin CT}

As an independent CT examination unit, the mobile cabin emergency CT consists of CT equipment, operation room, scanning room, air disinfection unit, radiation protection, network system, air conditioner, power distribution system, and a ventilation system. Compared with conventional CT, no on-site installation and debugging are required for the cabin CT. Because the equipment has been installed and debugged before delivery (in the factory), it can be quickly dispatched and set-up in various sites, including outdoors, and immediately used when powered on. It can avoid cross-infection with other patients in the hospital to achieve adequate disinfection, isolation, and protection. The operation and scanning rooms of the mobile cabin emergency CT are separate entities and kept isolated, which enables implementation of individualized and stepby-step disinfection protocols. The intelligent positioning of the CT examination eliminates the requirement for medical technicians to unnecessarily enter the scanning area, thereby preventing cross-infection between medical staff and patients.

The maneuverability, flexibility, effectiveness, and networking of the mobile cabin CT also make it of great applications in other emergency events, such as during earthquakes, flood relief operations, etc.

Imaging as a means of rapid screening against major national infectious diseases

A rapid and effective defense against infectious diseases, such as the novel coronavirus pneumonia (NCP) and atypical pneumonia, involves early detection, early diagnosis, early isolation, and early treatment. Imagingbased diagnosis is one of the essential methods for early detection. Moreover, early X-ray examination of NCP is often associated with high incidence of misdiagnosis rate, as no evidentiary abnormalities in the early stages of the disease can be detected. So far, the imaging-based diagnosis by fever clinics in most hospitals have depended mainly on digital radiography (DR). Whereas, CT is rarely employed as a separate screening modality. For patients with suspected NCP and undergoing CT examinations, it is a common challenge to avoid cross-infection if the Radiology department fails to achieve effective isolation and disinfection. In contrast, the mobile cabin CT can be quickly set up at a suitable site, including outdoors, with disinfection, isolation, and protection achieved as required. As a CT examination unit, the mobile cabin CT 
is particularly appropriate for the early detection of NCP patients in the portable cabin hospital.

The early CT manifestations of NCP are usually accompanied by ground glass shadow with or without interlobular septal thickening on the lateral field of the lung (8-10). Since the earliest manifestations of NCP are not often accurately captured by radiograph, a high-resolution CT (HRCT) of the chest is the primary tool for imagingbased screening and accurate diagnosis. The mobile cabin CT, equipped with 16-row detectors, 64-row detectors, or above, are equipped with a low dose, voice communication, and intelligent isolation positioning system to achieve remote positioning, especially suitable for the early screening and diagnosis of COVID-19 (Figures 6-8).

In addition to the screening of NCP, the mobile cabin $\mathrm{CT}$ is easily applicable to screen other infectious diseases.

\section{Applicability in earthquakes, major traffic accidents, and flood relief operations}

Natural disasters (such as earthquakes, typhoons, tsunamis, flash floods, mudslides, landslides, etc.), major traffic accidents, and the sudden collapse of buildings can inflict significant harm and devastation on communities. Timely delivery of on-site rescue, diagnosis, and treatment strategies are particularly important. At the moment of natural disasters, when in a remote area, or a traffic jam, or a large number of wounded are waiting to be treated, on-site medical rescue is challenging. The maneuverability, flexibility, and networking of the mobile cabin CT can play a vital role in sudden disasters. After placing the mobile cabin CT on a vehicle, it turns into an on-board CT, which can be delivered to the affected areas requiring CT examination.

(I) Fracture: the rapid and large-coverage scanning rendered by the mobile cabin CT can reveal the bone structure of all parts of the body in a single examination and diagnose a fracture, the precise location of the fracture, the fracture severity, and joint damage, etc. The three-dimensional reconstruction can pinpoint the injuries to the bone and joint (11).

(II) Cranial-cerebral trauma: on-site mobile cabin CT scan is the most effective and immediate examination for timely diagnosis of cranial-cerebral trauma, hematoma, skull fracture, etc.

(III) Multiple organs damage: the on-site mobile cabin
CT can be used to rapidly scan the thoracoabdominal region to detect whether there is any damage to the thoracoabdominal visceral organs in a single scan. It can be deployed to promptly assess traumatic features such as pneumothorax, rupture of liver or spleen, hematoma, bleeding, etc., which would be critical for rendering life-saving interventions.

\section{"One-stop" examination for stroke}

Stroke remains the leading causes of death or disability worldwide. As such, prompt diagnosis and treatment of stroke is crucial for saving lives and reducing disability. Stroke centers established in hospitals play an important role in the timely diagnosis and treatment of various types of stroke. Nonetheless, it is a pressing challenge to make an accurate diagnosis and provide treatment for stroke patients in remote areas of China owing to the vast territorial expanse, large population, and uneven medical resource allocation. Cerebral infarction and hemorrhagic strokes share similar acute onset and clinical manifestations. However, the treatment options of these two diseases are strikingly different and, at times, opposite to each other. Moreover, the optimal time window for thrombolytic therapy of cerebral infarction is within 6 hours, otherwise the risk of mortality and disability will greatly increase (12). The mobile cabin CT can offer oncall service, when accompanied by an ambulance it can provide CT examination for stroke patients on-site. Prompt identification of cerebral infarction and brain hemorrhage and timely delivery of appropriate treatment modalities can be carried out at the scene and on route to the hospital, which can help significantly increase the chances of survival and minimize the pathophysiological damage following stroke (12).

\section{Serving general health and responding to the aging health}

Low dose CT lung screening: Along with the improvement of living standards, healthcheck is a primary method for early diagnosis and treatment of important diseases (13). In particular, it relates to lung cancer, a major cause of death in the elderly population, where accurate diagnosis and treatment can save lives and reduce disability. Low-dose CT lung screening is the most effective measure for the early diagnosis of lung cancer. Unfortunately, due to the 

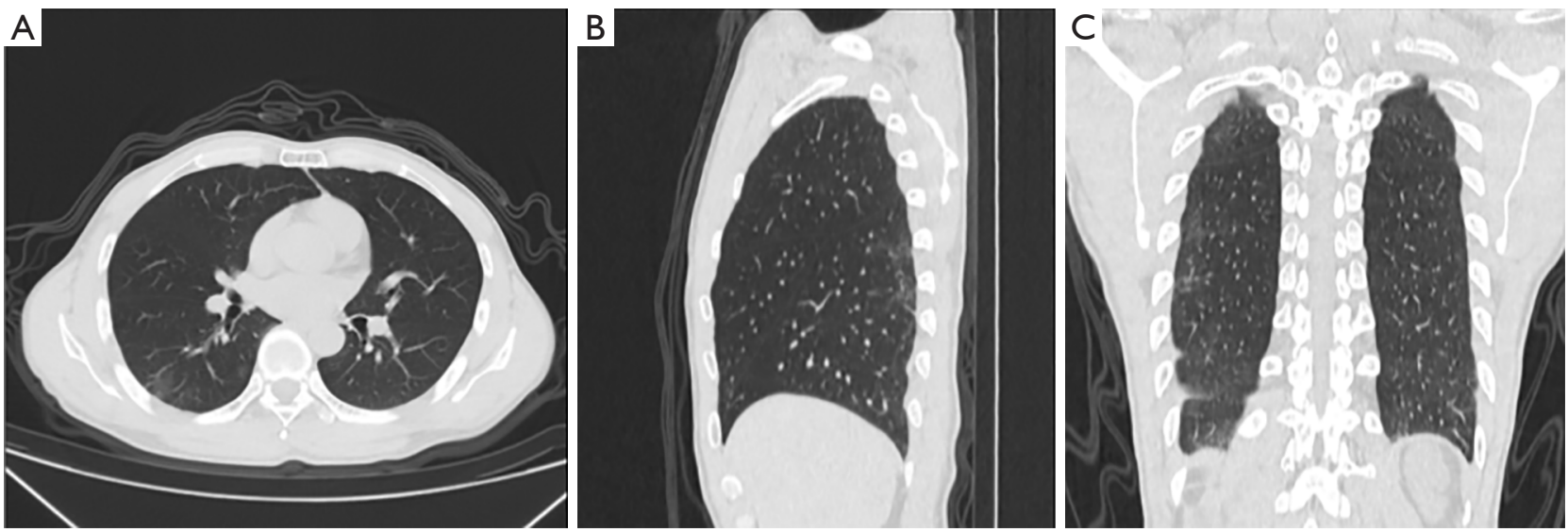

Figure 6 An early case of COVID-19, who manifests ground glass shadow under the pleura of the right lower lung on CT (pictures from the mobile cabin CT of Wuhan Optics Valley mobile cabin hospital).
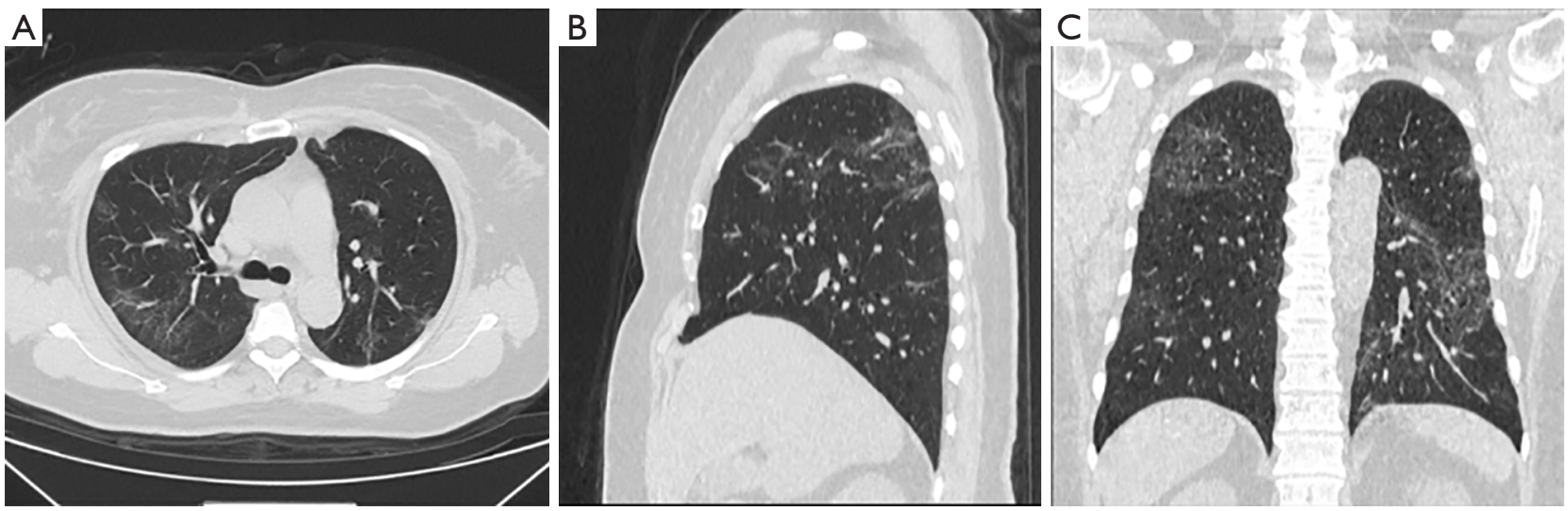

Figure 7 An early case of COVID-19, who presents multiple ground glass shadows and subtle mesh signs in the periphery of bilateral lungs on CT (pictures from the mobile cabin CT of Wuhan Optics Valley mobile cabin hospital).


Figure 8 A low dose CT scan $(120 \mathrm{kV}, 35 \mathrm{mAs})$, which reveals multiple ground glass shadows and vascular bundle thickening in the periphery of bilateral lungs. Thin-slice reconstruction at $1 \mathrm{~mm}$ (the right panel) shows air bronchus sign (pictures from the mobile cabin CT of Wuhan Optics Valley mobile cabin hospital). 
vast territory, large population and uneven medical resource allocation, it is not convenient for many people in remote areas of China to get access to low-dose CT lung screening. The maneuverability, flexibility, and networking of mobile cabin CT lent it possible to be delivered to remote areas to carry out on-site CT examination in remote areas, especially for the elderly.

(I) Cardiovascular and cerebrovascular disease screening: Equipped with 64- or 128-slice CT, the mobile cabin CT significantly improves the scan speed of CT examination, which can apply to the angiography of all parts of the body, including the coronary artery. The high-end image post-processing workstation enables rapid and simultaneous vasculature post-processing and reconstruction.

(II) In addition to CT, the mobile cabin CT can accomplish configuration expansion, with potential addition and integration of DR, mammography, or ultrasound, which makes it to a small and mobile Radiology department. This mobile cabin CT is especially suitable for mobile medical examinations in remote areas without proper health coverage.

\section{Enhanced configuration of national or provincial emergency medical rescue teams}

There are currently numerous well-equipped and mobilized national and provincial emergency medical rescue teams throughout China. For instance, the national emergency medical rescue team in Zhejiang Province is equipped with an operation vehicle, an inspection vehicle, a DR vehicle, an ultrasound vehicle, a power supply vehicle, a logistics support vehicle, a command vehicle, etc., but without a CT scanner. The use of CT diagnostic equipment is urgently needed for the prevention and control of respiratory infectious diseases, particularly, during emergency disaster relief operations. The mobile cabin CT can meet these demands. In the future, mobile cabin CT or on-board CT should become a standard configuration of national emergency medical rescue teams throughout the country.

\section{Supplement for the lack of CT configuration in rural hospitals}

The CT configuration of rural hospitals in China is insufficient, but the demand is ever increasing. For routine healthcheck examinations, many important CT examinations are often abandoned due to high demand, lack of time, and other reasons, which inevitably delay accurate disease diagnosis. Taking advantage of the flexibility of the mobile cabin CT, an appointment system can be adopted for non-emergency CT examinations in rural hospitals, and regular CT examinations can be incorporated into the routine health care of a given region. This would enable a full use of medical resources, solve the problem of insufficient CT configuration in rural hospitals, and reduce the waste of medical resources.

\section{Application in wartime}

Portable cabin hospital is an integral part of the military field mobile medical system, which mainly relies on a complete set of equipment to perform the medical care tasks for the wounded in the field. The most important characteristic of the portable cabin hospital is mobility. In addition, the modular equipment ensures not only complete medical treatment function, but also allows rapid expansion as needed. Thanks to its mobility, flexibility the shelter-type emergency CT employs low dose of radiation to render highresolution images in an intelligent and isolated environment connected to a network. It can be used as a module in the shelter hospital during wartime rescue efforts. Upon arrival at the scene, a full-body CT examination for the wounded and sick can be performed, followed by appropriate diagnosis and treatment of the injuries as soon as possible, which will increase the chances of survival and reduce disability.

\section{Emergency reserve}

As an important means of epidemic prevention and control, the mobile cabin CT can be integrated into the Radiology departments of hospitals to continue its function after the disaster. It can be activated into a mobile cabin CT at any time when the epidemic prevention and control and disaster emergency require so. As a reserve resource for epidemic prevention and control or emergency medical support, the mobile cabin CT possesses high flexibility, economic value, and practicability.

\section{Radiation protection for using mobile cabin CT}

The emergency CT rooms of the cabin unit are inspected 
for radiation leakage and the results meet the China national standards before installation. No additional protective measures are required for staff operating in compartments.

Machine protection: the air radiation count rate should be lower than $2.5 \mu \mathrm{Sv} / \mathrm{h}$ at $0.3 \mathrm{~m}$ from the external surface of the machine room, according to GB18871-2002, GBZ 165-2012, and GBZ 130-2013 standards. The room has been monitored in the factory by qualified institutions on radiation protection to ensure that the results meet the standards (14).

Medical staff protection: when the technician is in the control room with isolation controlling mode, no additional protective measures are required; If medical staff accompany a patient in machine room during the CT scan, they are required to wear lead clothing, lead apron, lead neckerchief and lead hat.

Patient protection: the patient's glands and organs adjacent to the scan area must be equipped with lead shielding apparel. If there are accompanying persons in the machine room, they too are required to wear lead clothing, lead apron, lead collar, and lead hat.

\section{Prospects for AI application into mobile cabin CT}

As an intelligent CT system, the mobile cabin CT offers a new paradigm by empowering the CT devices with AI technology. AI technology is deeply integrated into the whole CT data flow and workflow, which can significantly reduce radiation dose, improve image quality, optimize the scanning process, and greatly reduce the workload of doctors/technicians and improve diagnostic accuracy (15). Given the uneven distribution of medical resources in China and the severe shortage of imaging technicians and doctors in certain communities, AI technology will be crucial for a new intelligent workflow, reduce the training requirements and labor time of technicians, and decrease the error rate of manual/daily work. AI will also greatly reduce the doctor's reading time through various intelligent auxiliary diagnostic modules and assist him/her in making a more accurate diagnosis. For hospitals which lack radiologists or the proper means for accurate diagnosis, remote auxiliary module can be used for real-time uploading of images, intelligent diagnosis using cloud platform, and remote diagnosis/consultation, thereby reducing patients' waiting time for diagnosis results and speeding up the process of diagnosis. Images could also be uploaded to PACS of radiology departments or remote consultation centers, which can meet various needs of the application at any time, especially for a public emergency, earthquake relief, and field rescue, and so on.

The AI module accompanying the Mobile cabin CT consists of three subsystems, including a low-dose intelligent image reconstruction system, intelligent workflow, and computer-aided diagnosis. Low-dose intelligent reconstruction system is a double low-dose technology. On the one hand, it can automatically control the tube's output to effectively reduce the dose of radiation (which is approximately $30 \%$ lower than usual for the same quality of images) according to the body shape of the patient and the location of the scan. On the other hand, the use of NDI micro-dose iteration- "dual-domain" iterative algorithm can significantly reduce the dosage required for a CT scan, which in some cases is up to $45 \%$ lower than average on the typical lung scan condition (16). This technology is particularly suitable for current early diagnosis of COVID-19 infection as well as other scenarios of several CT scans in a short period for avoiding radiation damage or potential risk of carcinogenicity. The intelligent workflow uses AI algorithms to optimize clinical protocol individually, chooses parameters for scanning and reconstructing automatically, develops intelligent positioning, locating, image quality control and other functions during the scanning process and monitors the performance of devices intelligently and optimizes daily calibration process. The intelligent computer-aided diagnosis module uses the cloud platform for the remote computing and administration of big data to solve the existing problem associated with uneven distribution of medical resources and the lack of radiologists in some remote parts of the country, which would greatly reduce the workload of doctors and improve the accuracy of diagnosis.

\section{Emergency mobile cabin CT infection control}

\section{Infection control of emergency mobile cabin CT equipment}

Cleaning and disinfection of the emergency mobile cabin CT prior to use

Inspect the hygienic condition of the surface of CT equipment, keep the room tidy and clean, and replace used ones with new disposable sheets.

\section{Cleaning and disinfection of the emergency mobile cabin CT after use}

After the CT equipment is used, the equipment and the 


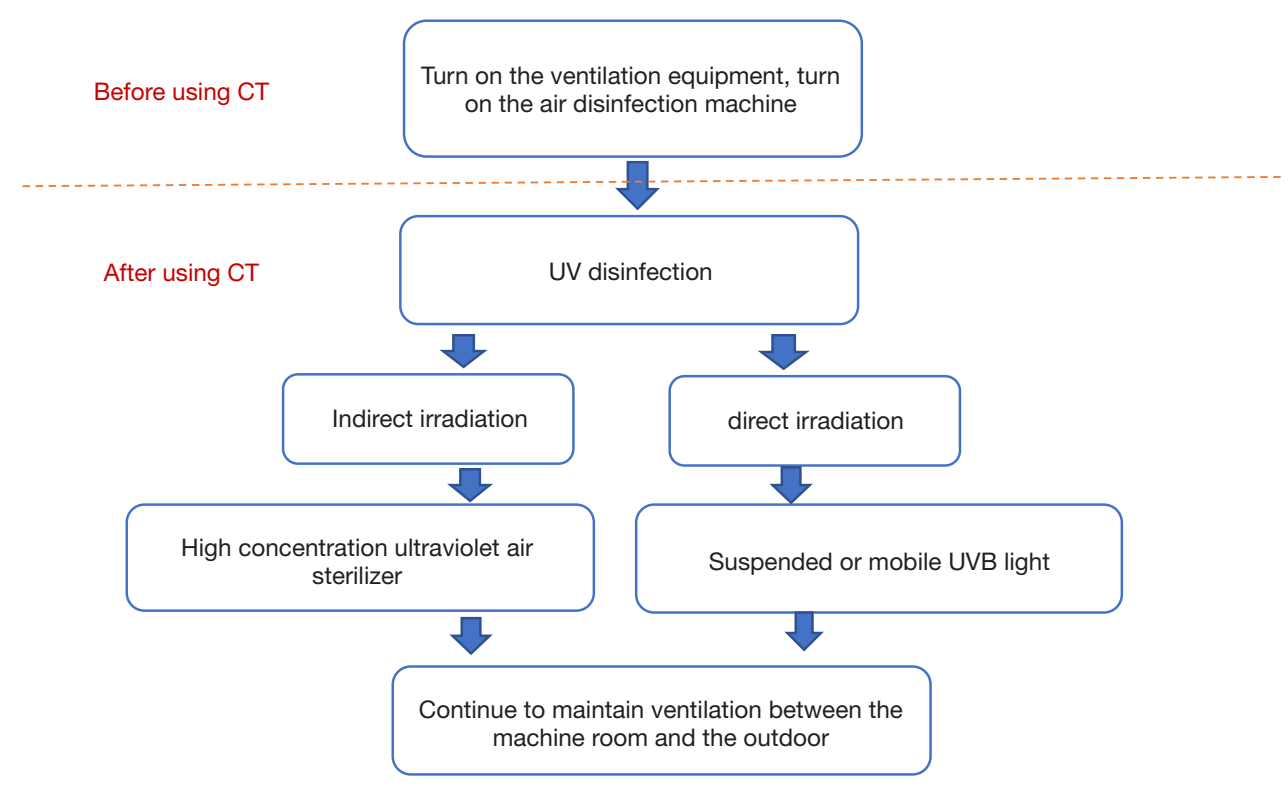

Figure 9 The cleaning and disinfection procedures of the emergency CT machine room in the square cabin.

computer room must be thoroughly cleaned and disinfected to avoid potential health and safety hazards to the medical staff and patients. When inspecting CT equipment, if there is visible dirt on the surfaces of the equipment, use a disposable absorbent material. When needed, wipe the surface of the equipment with gauze dipped in $75 \%$ ethanol. The surfaces that the patient may have contacted need to be wiped. Remove the ground trash and debris, and use 2,000 mg/L chlorine disinfectant to wipe and disinfect the ground (17).

Imaging equipment cleaning and disinfection program: the equipment room should be thoroughly cleaned first. Do not use cleaning materials other than soap and water to clean the surfaces of the system. Do not use detergents or organic solvents to clean the CT system because potent cleaning agents, ethanol, and organic cleaning agents may damage the surface gloss of the equipment and weaken the structural strength. Disinfection should be carried out by following the recommendations of the CDC (http://www.cdc.gov/ hicpac/pdf/guidelines/disinfection_nov_2008.pdf) for lowand medium-level disinfection (18). The following materials can be used for cleaning and disinfection: (I) Spray cleaner or wipes equivalent to bleach (concentration less than $10 \%$ ); (II) low-level or medium-level disinfection wipes or liquid; (III) 3\% hydrogen peroxide; (IV) Ethanol; (V) Desulfurized liquefied petroleum gas; (VI) Quaternary ammonium compound; (VII) Benzyl-C12-18-alkyl dimethyl; (VIII) Salt with 1,2-Phenylisothiazole-3 (2H) -one 1,1-dioxide(1:1); (IX) distilled water; $(X)$ industrial alcohol.

Pay particular attention to the cleaning procedures of the device: (I) When cleaning the front and back covers of the scanner, please cover the microphone to avoid the leakage of the cleaning agent. (II) Care must be taken when cleaning the buttons and inside the holes of the rack to avoid the penetration of the cleaning fluid into the rack. (III) Blood and contrast agents may carry health risks. When removing blood or residual contrast agent, appropriate safety precautions should be taken. (IV) It is not recommended to use spray disinfection tools. (V) It is strictly forbidden to use flammable or explosive disinfectant sprays. (VI) It is not recommended to use high-concentrations of chlorine-containing disinfection solution for machine-room disinfection.

\section{Control of air born infection in the emergency mobile cabin CT computer room}

\section{Air purification and disinfection of the machine room before the use of emergency $\mathrm{CT}$}

Turn on the ventilation equipment, keep the air in the 
equipment room in constant exchange with the outside, and turn on the air disinfection machine.

Air purification and disinfection of the machine room after the use of emergency CT

The computer room can be sterilized by indirect ultraviolet irradiation and direct irradiation. Indirect irradiation method: High-intensity ultraviolet air sterilizer, general disinfection can be achieved after 30 minutes of exposure. Direct irradiation method: With the room devoid of personnel and patients, UV lamp suspension type or mobile type direct irradiation can be adopted. When using indoor suspended UV disinfection, the number of UV disinfection lamps $(30 \mathrm{~W}$ UV lamp, intensity $>70 \mu \mathrm{W} / \mathrm{cm}^{2}$ at 1.0 meter) installed indoors is an average of not less than $1.5 \mathrm{~W}$ per cubic meter (power) exposure time in $30 \mathrm{~min}$. After the work is completed and after the room is evacuated, turn on the UV lamp (30 $\mathrm{W} / 16 \mathrm{~m}^{2}$ ) to irradiate and disinfect for more than 30 minutes, while continuing to maintain the ventilation of the computer room (with the outdoor air).

\section{Cleaning and disinfection procedure of emergency mobile cabin CT machine room}

Turn on the ventilation equipment, keep the air in the equipment room in constant exchange with the outside, turn on the CT indoor ultraviolet circulation air disinfection machine, and follow the operating instructions to ensure regular operation. The cleaning and disinfection procedures of the emergency CT machine room in the square cabin are shown in the following figure (Figure 9).

\section{Infection control of medical technicians}

\section{Disinfection and isolation of emergency CT operated by medical technicians}

Depending on the type/purpose of CT examination and the clinical diagnosis of patients, the medical technicians are required to carry out appropriate disinfection and isolation procedures when operating the emergency cabin CT. The detailed cleaning and disinfection procedures are shown in Figure 10.

\section{Disinfection and isolation of CT examination for common fever patients}

Most patients are expected to present with symptoms of ordinary fever, and some may be suspected to have been infected with COVID-19. Therefore, the radiologist should make a timely diagnosis, report the suspected infection of COVID-19 in a timely fashion, and disinfect and isolate the unit immediately thereafter.

(I) The operation technician adopts secondary protection measures. Wear disposable working caps, N95 medical protective masks, goggles or protective screens, disposable latex gloves, and wear medical protective clothing and disposable shoe covers (19). Strictly implement hand hygiene.

(II) Before the examination, the whole CT examination bed is covered with a disposable sheet, and after examination the disposable sheet is replaced.

(III) If the patient can cooperate, use the voice control to direct the patient to lie on the examination table and position properly. If the CT has an automatic positioning function, automatic positioning can be used.

(IV) In the case of severely ill patients, an accompanying person or technician (with tertiary protection) is required to enter the machine room to assist in positioning, and the technician must not return to the control room without proper disinfection.

(V) After the CT technician completes the inspection, the hands are disinfected immediately.

(VI) In case of contact with suspected cases, protective clothing should be changed between each patient's contact.

(VII) Once a suspected infection with new coronavirus pneumonia is found, the computer room should be disinfected before use.

Disinfection and isolation of CT examination in cases of suspected infection and confirmed diagnosis of COVID-19

The operation technician adopts tertiary protection $(20,21)$. Based on the second-level protection, wear protective masks, goggles, or positive pressure headgear. Pay attention to wear protective clothing as required by the hospital infection department. Strictly implement hand hygiene.

(I) Before the examination, place a disposable sheet to cover the entire CT examination bed.

(II) If the patient can cooperate, the technician will execute the entire scan from the control room only. In this case, the patient and the technician enter through the scanning room door and the operating room door, respectively. Voice control directs the patient to lie down on the examination table and position himself/ 
Disinfection and isolation procedures for medical technicians performing operations

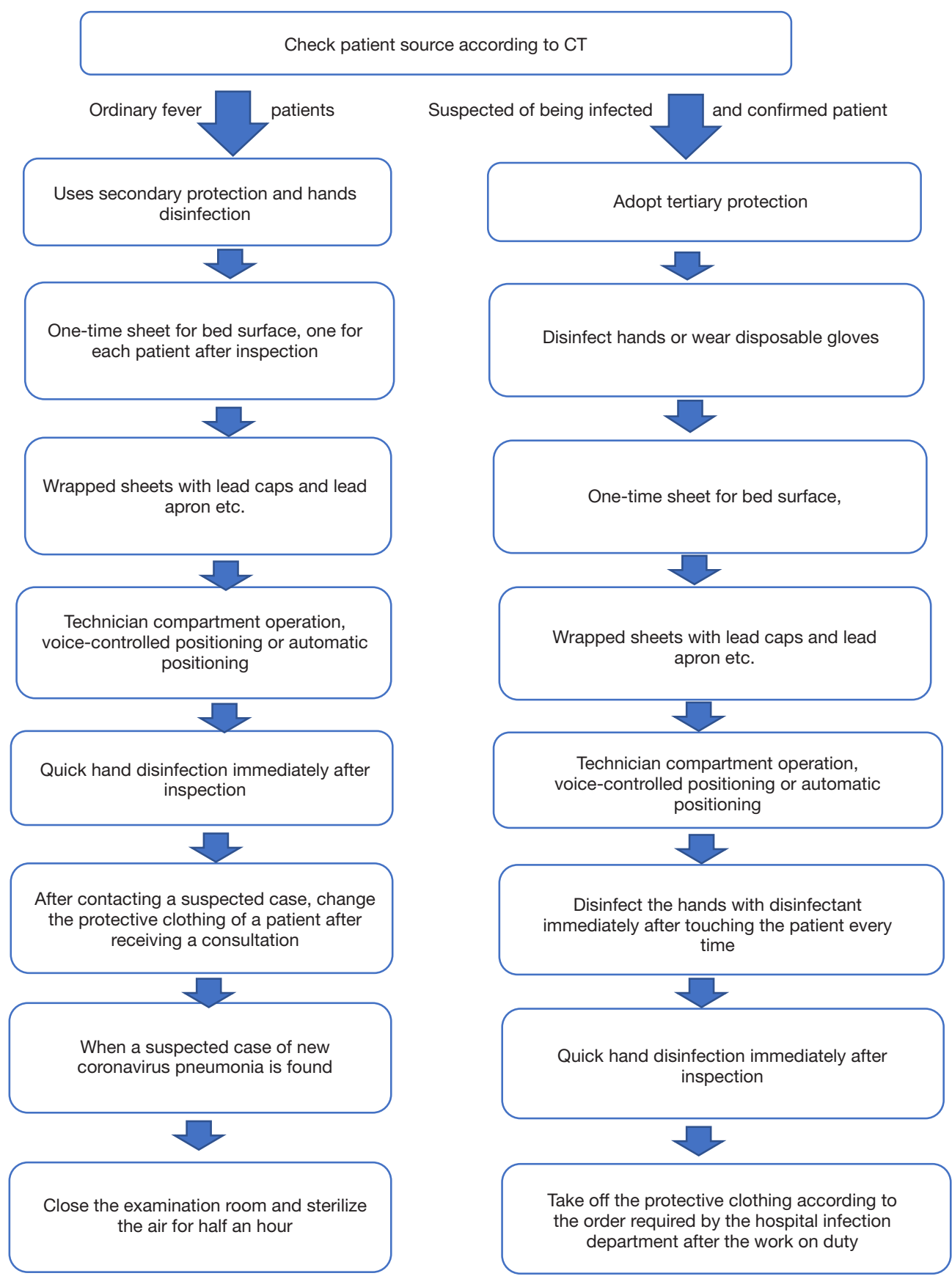

Figure 10 Medical technicians operating emergency mobile cabin CT: cleaning and disinfection procedures. 
herself. If CT has an automatic positioning function, automatic positioning can be adopted.

(III) In the case of severely ill patients, an accompanying person or technician (with tertiary protection) is required to enter the machine room to assist in positioning, and the technician must not return to the control room without thorough disinfection.

(IV) Disinfect hands with quick-drying disinfectant immediately after touching each patient.

(V) For patients with suspected infection or with confirmed cases with COVID-19, they are not required to replace their protective clothing after the first CT scan if they have continuous inspection.

(VI) After the CT technician completes the examination, his/her hands must be disinfected immediately.

(VII) At the end of the work shift, medical staff take off and dispose the protective clothing as required by the hospital.

The detailed cleaning and disinfection procedures are shown in Figure 10.

\section{Patient infection control}

Disinfection and isolation procedures for patients undergoing CT examination

CT examination of common fever patients

(I) Patients including accompanying staff should wear medical surgical masks throughout the CT examination procedure;

(II) Before entering the scanning room, use a disinfectant to sterilize hands;

(III) Use a disposable bed sheet for CT examination;

(IV) Protective equipment, such as lead caps and lead aprons, should be wrapped with disposable sheets and isolated from the patient's body and clothing;

(V) Use voice control as much as possible when giving the examination instructions. If the technician must be in contact with the patient, try to keep a distance of more than $1.5 \mathrm{~m}$;

(VI) Patients who have been examined should be separated from each other to avoid conversation and close contact (22);

(VII) Patients (including accompanying individuals) must enter and exit through the door of the scanning room and are not allowed to move around without permission.

(VIII) Once a scanned patient is suspected of being infected with COVID-19, the computer room should be disinfected before re-use.

\section{CT examination of suspected infection and confirmed} diagnosis of the new coronavirus cases

Patients including accompanying persons must wear N95 medical protective masks throughout the CT examination procedure:

(I) Before entering the equipment room, use a disinfectant to sterilize hands or wear disposable gloves;

(II) Before the examination, place a disposable sheet to cover the entire CT examination bed;

(III) Protective equipment, such as lead caps and lead aprons, should be wrapped with disposable sheets and isolated from the patient's body and clothing;

(IV) Use voice control as much as possible when giving examination instructions. If the technician has to contact the patient, try to keep a certain distance;

(V) Patients (including accompanying personnel) must enter and exit through the machine room door;

(VI) Restrict the scope of activities for the patients and accompanying persons and do not allow them moving around without permission;

(VII) After the examination is completed, the patients return to the ward and do not stay at the CT site.

\section{Procedures for admission, consultation, and critical emergency condition}

During the epidemic period of infectious pneumonia, customized CT systems should be used to scan patients with fever or respiratory symptoms in fever clinics or emergency/ outpatient/wards in order to minimize the spread of infectious diseases and contain/control hospital crossinfection rates. Technicians involved in consultations should be informed of the full set of disinfection and protection protocols. CT scans must be carried out using intelligent positioning whenever possible to reduce contact between the technicians and patients. As shown in the figure below (Figure 11), after intelligent positioning in mobile cabin CT, the technician does not need to be in direct contact with the patients, which helps to reduce cross-infection between 


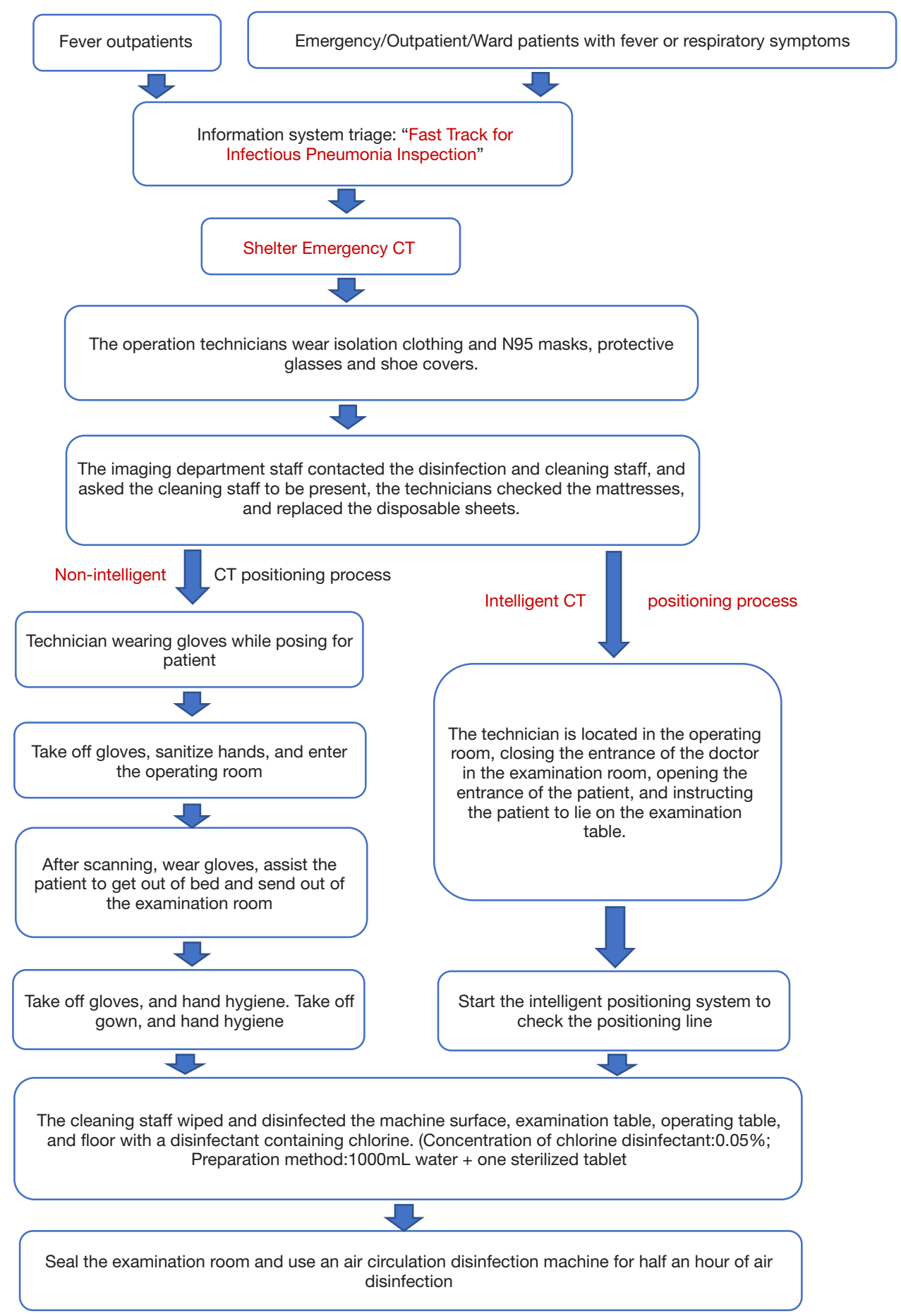

Figure 11 Procedures for admission, consultation, and critical emergency condition using intelligent or non-intelligent CT positioning. Whether to choose intelligent CT positioning depends on the CT equipment. We recommend intelligent CT positioning process if your CT machine with this function. 
health-care providers and patients.

\section{Acknowledgments}

We appreciate the contributions of Drs. Wenwen Gao, Lei Du, Yige Wang, Xiuxiu Liu, Yue Chen, Xiaowei Han from China-Japan Friendship Hospital, and Drs. Penghui Sun, Yuehong Liu, Chengxi Yan, Yingying Li from Beijing Chaoyang Hospital, who greatly assist in proofreading, spell checking and formatting.

Funding: This work were supported by National Nature Science Foundation of China (No. 62041601) and the Fundamental Research Funds for the Central Universities, Nanjing University (No. 2020-021414380462).

\section{Footnote}

Provenance and Peer Review: This article was a free submission to the editorial office, Quantitative Imaging in Medicine and Surgery. The article did not undergo external peer review.

Conflicts of Interest: All authors have completed the ICMJE uniform disclosure form (available at http://dx.doi. org/10.21037/qims-20-980). Prof. YXJW serves an unpaid Editors-in-Chief of Quantitative Imaging in Medicine and Surgery. The other authors have no conflicts of interest to declare.

Open Access Statement: This is an Open Access article distributed in accordance with the Creative Commons Attribution-NonCommercial-NoDerivs 4.0 International License (CC BY-NC-ND 4.0), which permits the noncommercial replication and distribution of the article with the strict proviso that no changes or edits are made and the original work is properly cited (including links to both the formal publication through the relevant DOI and the license). See: https://creativecommons.org/licenses/by-nc-nd/4.0/.

\section{References}

1. Chao Y, Shuai W, Su W, Liu S, Niu F, Tan S, Wu C, Duan D. Development of vibration damping device of CT shelter. China Medical Devices 2014;29:22-24+27.

2. Su W, Tan S, Chao Y, Niu F, Yang M, Shuai W, Su C, Duan D, Wu L. Study and Design of CT Shelter's Layout. Chinese Medical Equipment Journal 2013;34:29-30.

3. Kakinuma R, Ohmatsu H, Kaneko M, Kusumoto M,
Yoshida J, Nagai K, Nishiwaki Y, Kobayashi T, Tsuchiya R, Nishiyama H, Matsui E, Eguchi K, Moriyama N. Progression of focal pure ground-glass opacity detected by low-dose helical computed tomography screening for lung cancer. J Comput Assist Tomogr 2004;28:17-23.

4. Rotondo A, Guidi G, Catalano O, Smaltino F. High resolution computerized tomography in the study of the lung parenchyma. Possibility of a low-dose protocol. Radiol Med 1994;87:603-7.

5. Kalra MK, Maher MM, Toth TL, Schmidt B, Westerman BL, Morgan HT, Saini S. Techniques and applications of automatic tube current modulation for CT. Radiology 2004;233:649-57.

6. Dong X, Niu T, Zhu L. Combined iterative reconstruction and image-domain decomposition for dual energy CT using total-variation regularization. Med Phys 2014;41:051909.

7. Li L, Qin L, Xu Z, Yin Y, Wang X, Kong B, Bai J, Lu Y, Fang Z, Song Q, Cao K, Liu D, Wang G, Xu Q, Fang $\mathrm{X}$, Zhang S, Xia J, Xia J. Using Artificial Intelligence to Detect COVID-19 and Community-acquired Pneumonia Based on Pulmonary CT: Evaluation of the Diagnostic Accuracy. Radiology 2020;296:E65-E71.

8. Chung M, Bernheim A, Mei X, Zhang N, Huang M, Zeng X, Cui J, Xu W, Yang Y, Fayad ZA, Jacobi A, Li K, Li S, Shan H. CT Imaging Features of 2019 Novel Coronavirus (2019-nCoV). Radiology 2020;295:202-7.

9. Lei J, Li J, Li X, Qi X. CT Imaging of the 2019 Novel Coronavirus (2019-nCoV) Pneumonia. Radiology 2020;295:18.

10. Duan YN, Qin J. Pre- and Posttreatment Chest CT Findings: 2019 Novel Coronavirus (2019-nCoV) Pneumonia. Radiology 2020;295:21.

11. Novelline RA, Rhea JT, Rao PM, Stuk JL. Helical CT in emergency radiology. Radiology 1999;213:321-39.

12. Muir KW, Buchan A, von Kummer R, Rother J, Baron JC. Imaging of acute stroke. Lancet Neurol 2006;5:755-68.

13. Bach PB, Mirkin JN, Oliver TK, Azzoli CG, Berry DA, Brawley OW, Byers T, Colditz GA, Gould MK, Jett JR, Sabichi AL, Smith-Bindman R, Wood DE, Qaseem A, Detterbeck FC. Benefits and harms of CT screening for lung cancer: a systematic review. JAMA 2012;307:2418-29.

14. GB18871-2002. Basic standards for protection against ionizing radiation and for the safety of radiation sources. Available online: https://webstore.ansi.org/standards/spc/ gb188712002

15. Shiraishi J, Li Q, Appelbaum D, Doi K. Computer-aided diagnosis and artificial intelligence in clinical imaging. 
Semin Nucl Med 2011;41:449-62.

16. Lell MM, Wildberger JE, Alkadhi H, Damilakis J, Kachelriess M. Evolution in Computed Tomography: The Battle for Speed and Dose. Invest Radiol 2015;50:629-44.

17. Health Commission of the People's Republic of China. The standardization for sterilization technique $\sin$ hospital. Available online: http://www.nhc.gov.cn/wjw/ s9496/201204/54510.shtml. Published 2012. Accessed March 11, 2020.

18. Centers for Disease Control and Prevention. Guideline for Disinfection and Sterilization in Healthcare Facilities, 2008. Available online: https://www.cdc.gov/ infectioncontrol/guidelines/disinfection/Guideline

19. Huang Z, Zhao S, Li Z, Chen W, Zhao L, Deng L, Song B. The Battle Against Coronavirus Disease 2019 (COVID-19): Emergency Management and Infection

Cite this article as: Yang $\mathrm{Q}, \mathrm{Xu} \mathrm{H}$, Tang $\mathrm{X}$, Hu C, Wang $\mathrm{P}$, Wáng YXJ, Wang Y, Ma G, Zhang B; on behalf of the Expert Committee of Medical Imaging Engineering and Technology Branch of the Chinese Society of Biomedical Engineering. Medical Imaging Engineering and Technology Branch of the Chinese Society of Biomedical Engineering expert consensus on the application of Emergency Mobile Cabin CT. Quant Imaging Med Surg 2020;10(11):2191-2208. doi: 10.21037/ qims-20-980
Control in a Radiology Department. J Am Coll Radiol 2020;17:710-6.

20. Health Commission of the People's Republic of China. The guideline for prevention and control of hospital acquired infections of airborne pathogens. Available online: http://www.nhc.gov.cn/wjw/s9496/201701/7e0e8fc 6725843aabba8f841f2f585d2.shtml

21. Peng X, Xu X, Li Y, Cheng L, Zhou X, Ren B. Transmission routes of 2019-nCoV and controls in dental practice. Int J Oral Sci 2020;12:9.

22. Lai CC, Shih TP, Ko WC, Tang HJ, Hsueh PR. Severe acute respiratory syndrome coronavirus 2 (SARSCoV-2) and coronavirus disease-2019 (COVID-19): The epidemic and the challenges. Int J Antimicrob Agents 2020;55:105924. 